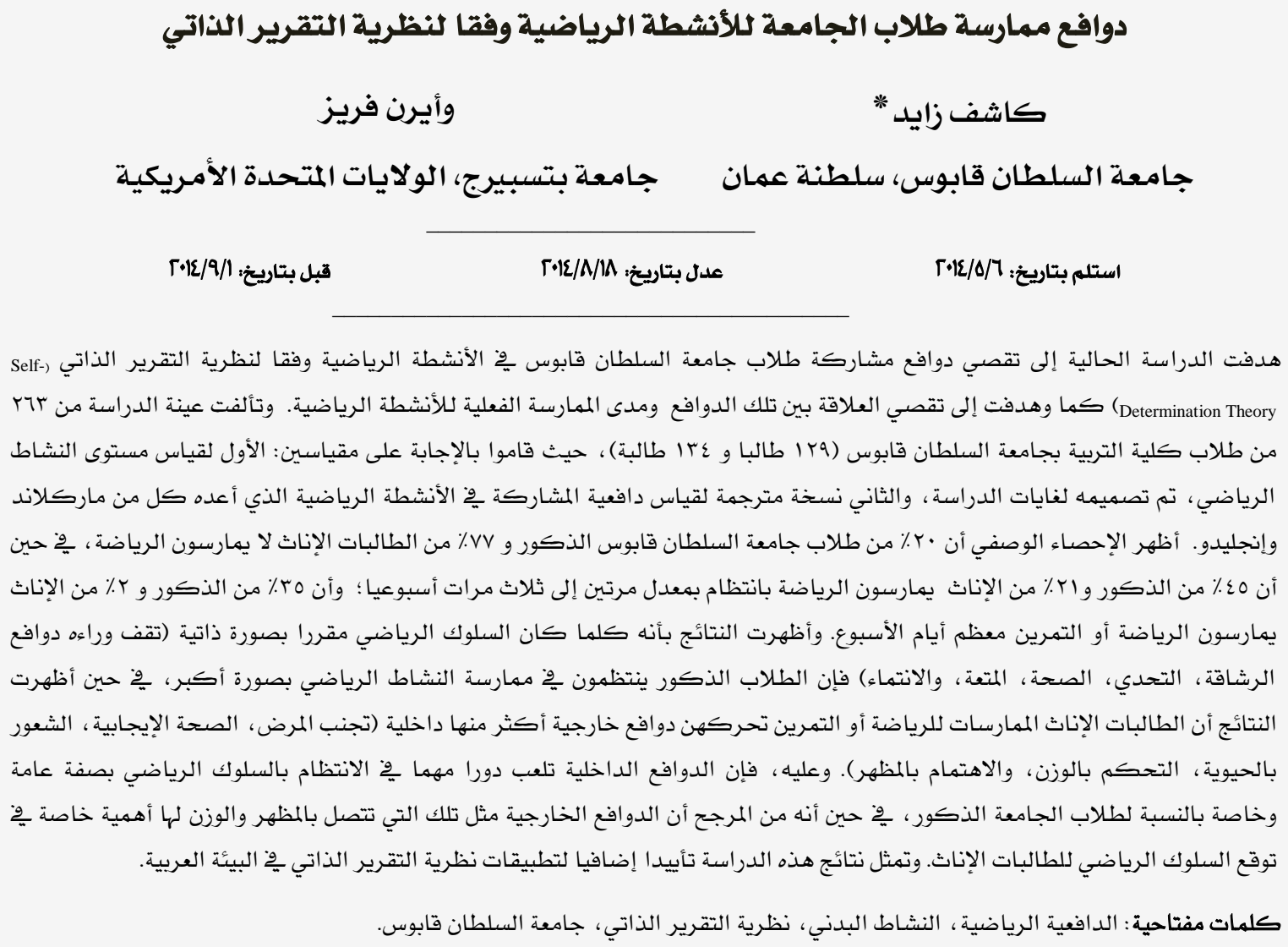

\title{
University Students' Motives to Exercise According to the Self-Determination Theory
}

Kashef Zayed*

Sultan Qaboos University, Sultanate of Oman
\& Irene Frieze

Pittsburgh University, United States

The present study investigated exercise behavior and its motives among Sultan Qaboos University students. Self-determination theory was used to investigate the relationship between these motives and the actual exercise behavior. The study sample consisted of 263 students from the Faculty of Education at Sultan Qaboos University (129 male and 134 female). The participants completed two questionnaires: an exercise behavior questionnaire which was developed specifically for this study; and an Arabic version of the Exercise Motivation Inventory (EMI-2), which was developed by Markland and Ingledew. Descriptive statistics showed that $20 \%$ of male students and $77 \%$ of female students didn't participate at all in any sport and exercise activities; $45 \%$ of male students and $21 \%$ of female students participated moderately in sport and exercise activities (2-3 times a week); while $35 \%$ of the male students and $2 \%$ of the female students participated intensively in sport and exercise activities (almost on daily basis). Results indicated that intrinsic motives for exercise behavior (challenge, revitalization, health, affiliation and enjoyment) were common among the male participants. However, physically active females, extrinsic motivations (ill-health avoidance, positive health, revitalization, weight management, and appearance) were the major driving forces for their exercise behavior. Results of the current study provide support for the application of the self-determination theory within the Arab context.

Keywords: Exercise motivation, physical activity, self-determination theory, Sultan Qaboos University.

*kashefz@squ.edu.om 
الخارجبة لأنه الخث عليه بأني من البيئة الخارجية. أما

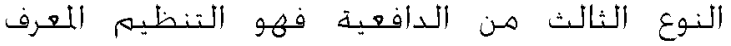
وهو قريب من الاختيار الذاتي (Identified Regulation)

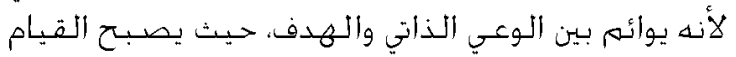

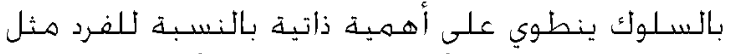

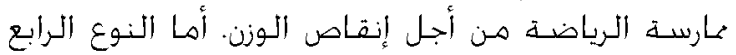

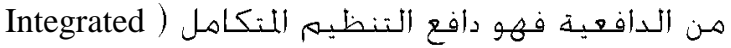
(Regulation

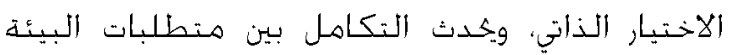

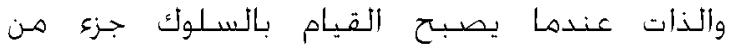

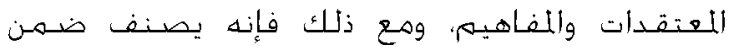

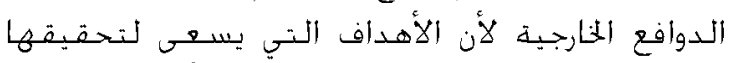

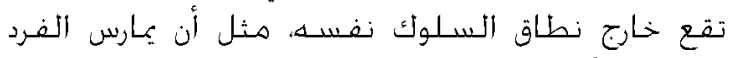

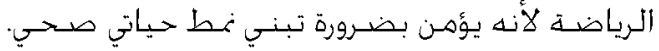
إن المبدأ الأساسي من نظريذ التقرير الذاتي لدى الفي

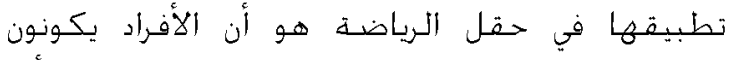

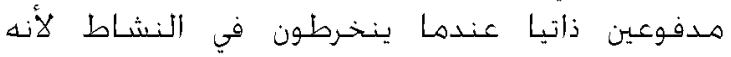

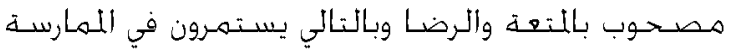

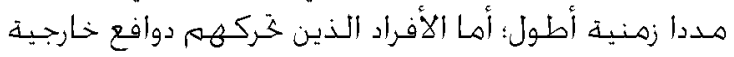

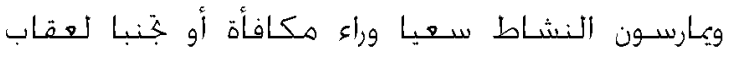

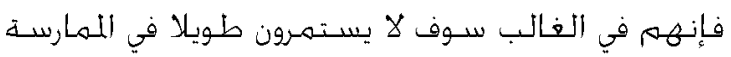
Ingledew \& Markland, 2008; Murcia, Roman, ) Galindo, Alonso, \& Gonzalez-Cutre, 2008

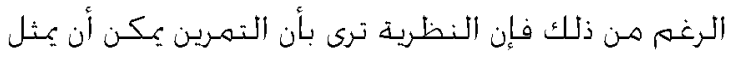

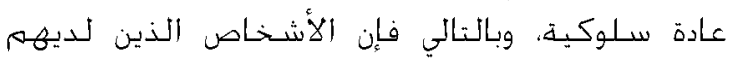

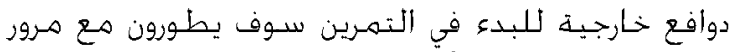

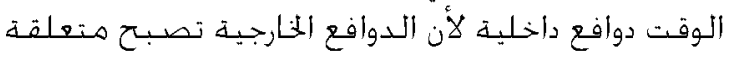

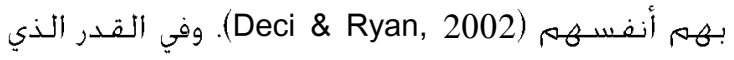
تنوافق فيه الدوافع الخارجيد مع الذات الذات واحتباجاتها

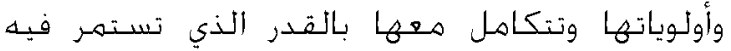
الممارسـة وتكون مثابة خبرات إيمابية.

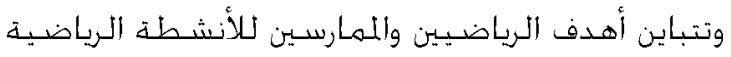

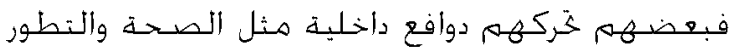

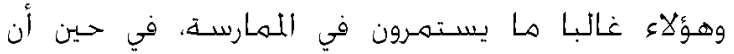

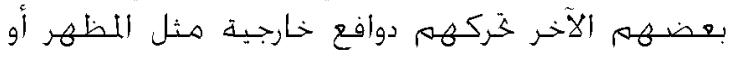

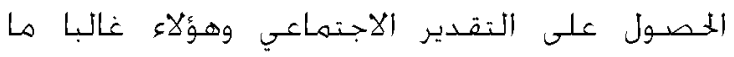

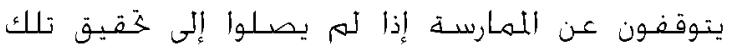
الأهداف (Ryan, Williams, Patrick, \& Deci, 2009). وترى نظرية التقرير الذاتي أن الدوافع المرتبطة بالنظهر

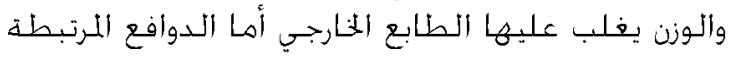

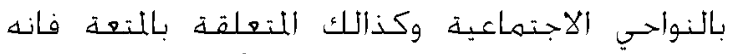

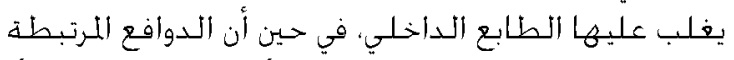

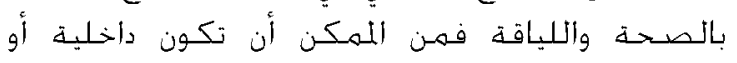
خارجية.
ترى نظرية التقرير الذاتي (Self-determination Theory)

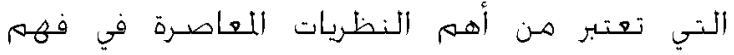
Lavalle, Williams, Kremer, and Moran, ) الدافعبة التعنية 2004) أن هنانك ثلاثة حاجات داخلبة رئبسية تلعب دورا

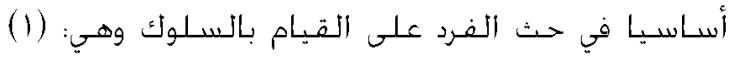

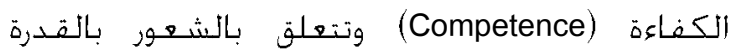

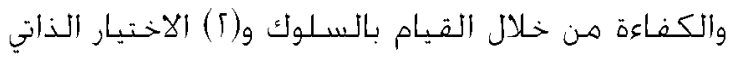

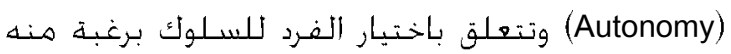

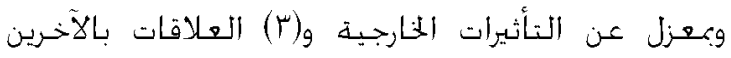
وتتعلق بمدى الإشباع الذي يوفره القيام (Relatedness)

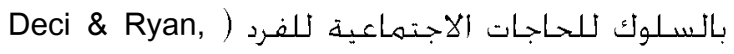

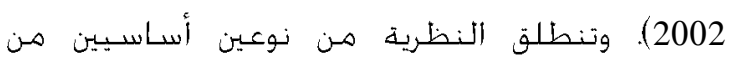

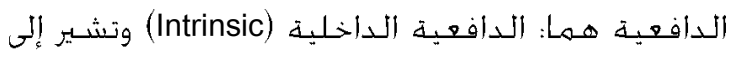

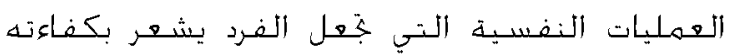

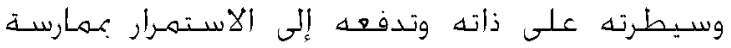

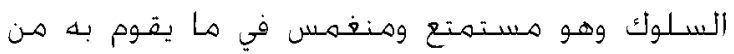
نشاط (Ryan \& Deci, 2002; Vallerand, 2001).

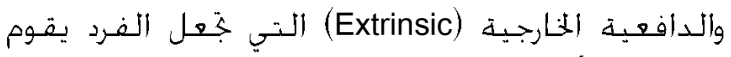

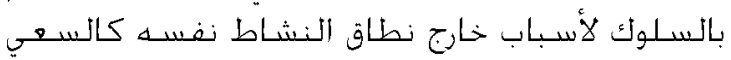

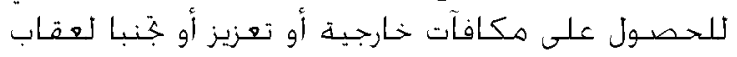
(Aallerand, 1997)

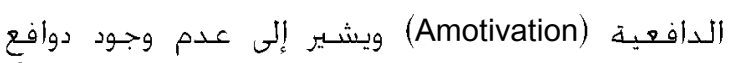

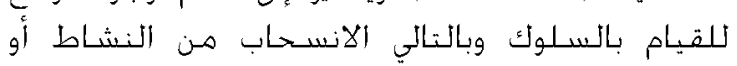
التوقف عن القيام به (Vallerand, 2001).

وقد طور كل من ديسي وريان (Deci \& Ryan,2002)

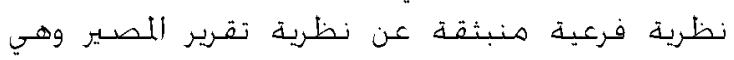

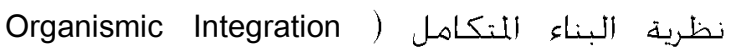

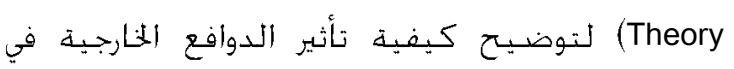
السـلوك. وتدرس هذه النظرية مدى إضفاء الصبغة الصنة

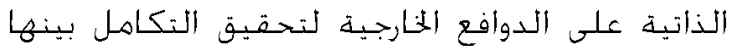

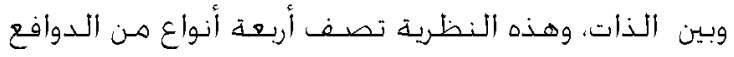

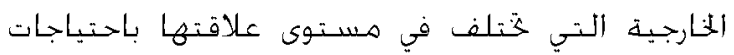

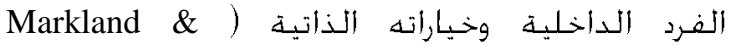
التجأ Ingledew, 2007; Deci \& Ryan, 2002

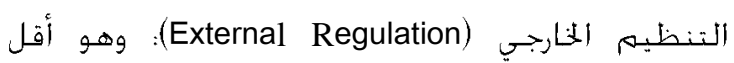

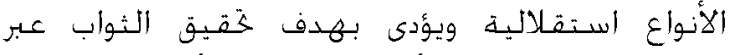

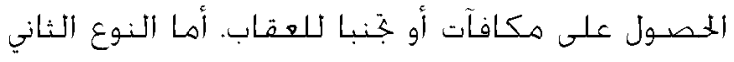

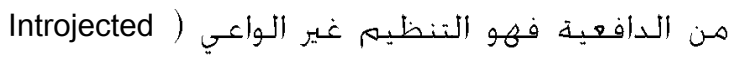
(Regulation

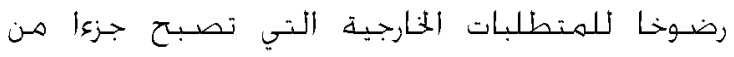

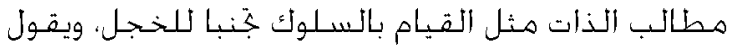

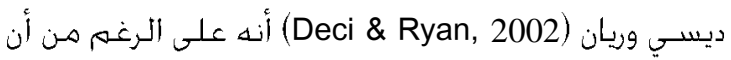

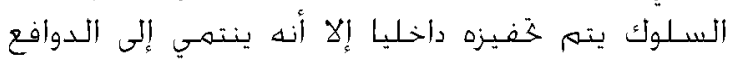


أن الدوافع الداخليذ يمكن تقويتها من خلال الثأثير على

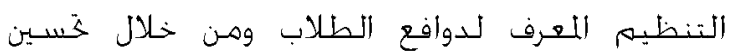
قدراتهم الرياضية التي تؤثر على إدراكهم لكفاءتهم.

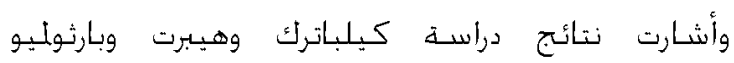
(Kilpatrick, Hebert, and Bartholomew, 2005)

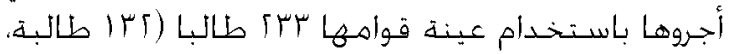

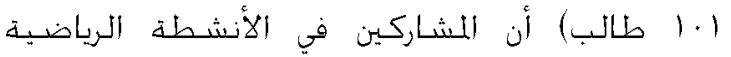

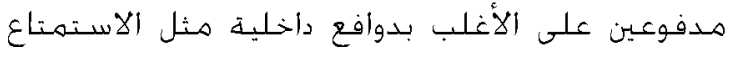

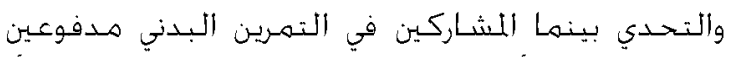

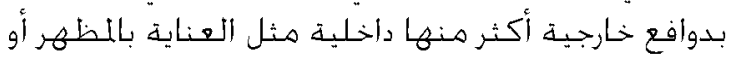

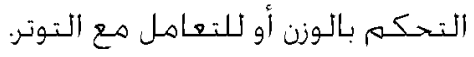

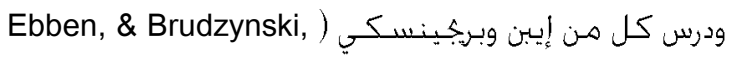

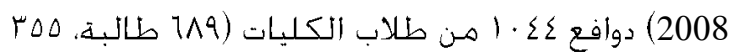

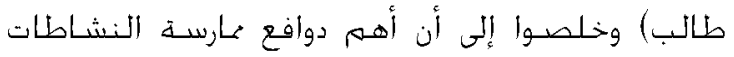

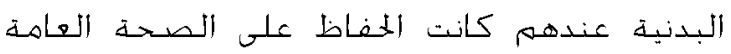

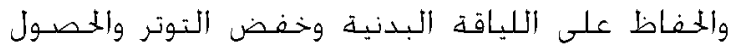

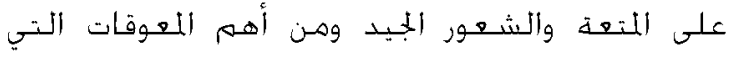

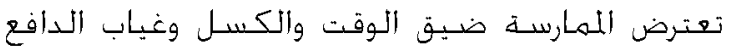
وعدم توفر الطاقة.

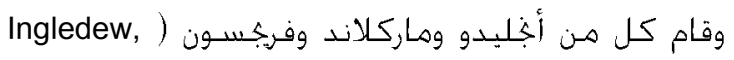
بدراسـة أشـاروا خلالاهـا

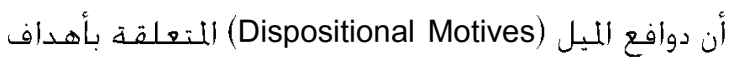
الفرد الحياتية بصورة عامة، بمعنى ما يريد وما لا لا بريد،

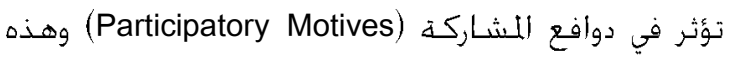

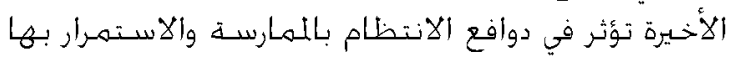
وبالتالي خدد نمط حياة الفرد. (Regulatory Motives) وفام كل من كافلو وسيرفيللو وجيمنيز وإيملاسيوس Cavlo, Cervello, Jimenez, Iglesias, \& ) وموريشيا هن بإجراء دراسـة لتقصي دوافع مارسـة (Murcia, 2010

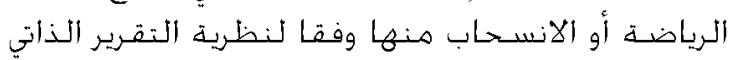

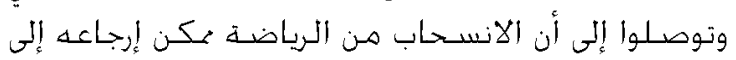

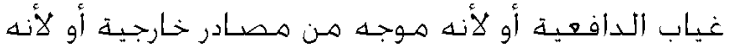
لم بنم اختياره ذاتيا أو أنه لا برتبط من بالعلاقة مع الآخربن.

وتقصى كل من دونكان وهول وويلسون وجبيني (Duncan, Hall, Wilson, \& Jenny, 2010 )

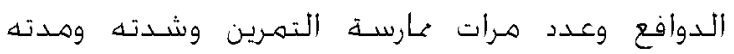

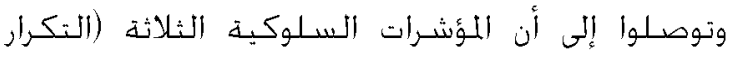

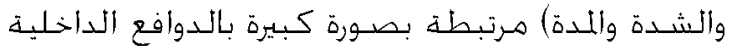

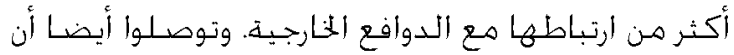

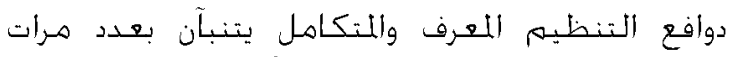

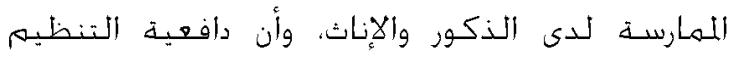

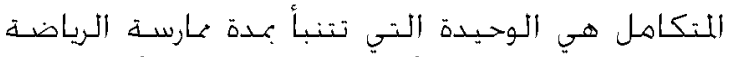

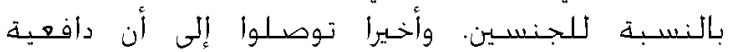

ووفقا لنظرية التقرير الذاتي فإن الاهنهام بالقيام

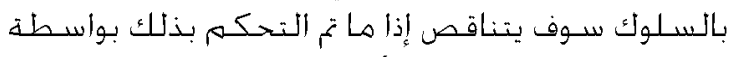

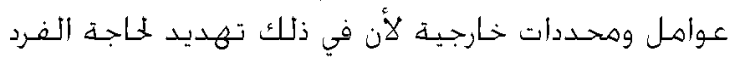

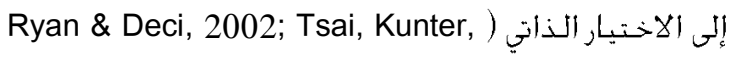
(Ludtke, Trautwein, and Ryan, 2000

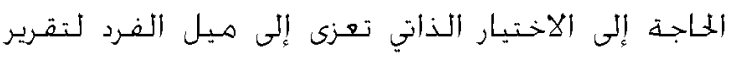

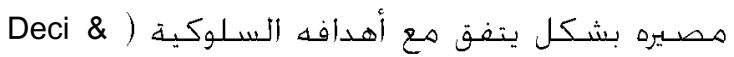

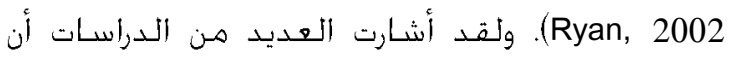

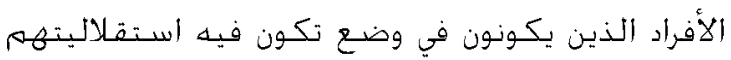

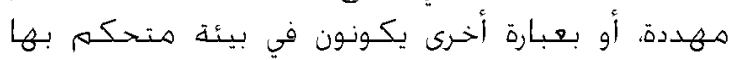

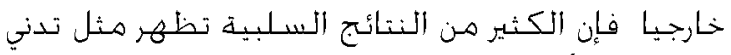

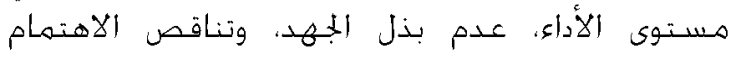

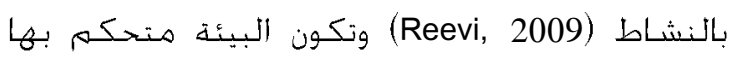

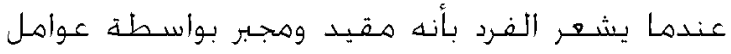

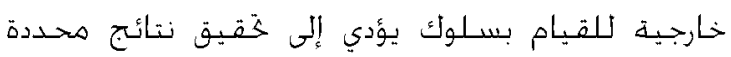

(Deci \& Ryan, 2002)

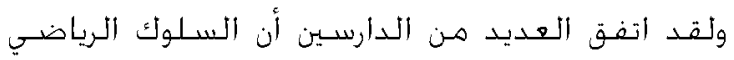

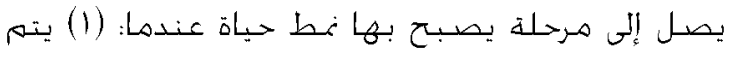

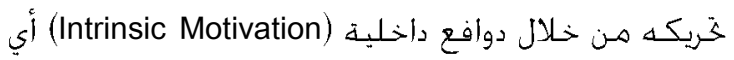

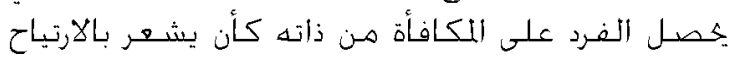

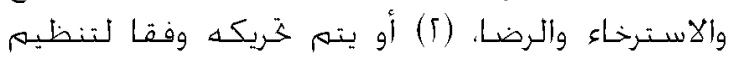

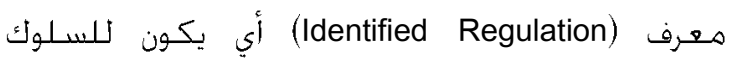

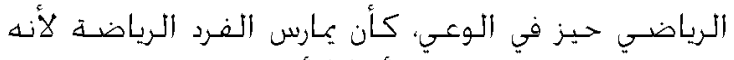

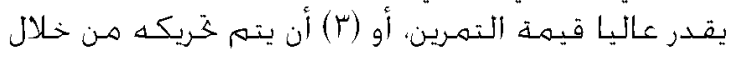

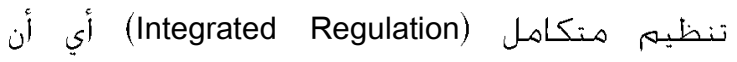
السـلوك الرياضي بتفق وينسجـم مع القيهم والمبادئ

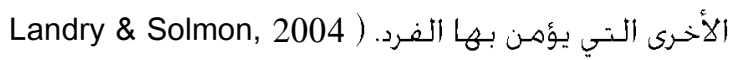
Wilson, Rodgers, Blanchard, \& Gessell, 2003; .(Wilson, Rodgers, \& Fraser, 2002

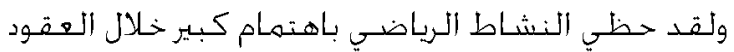

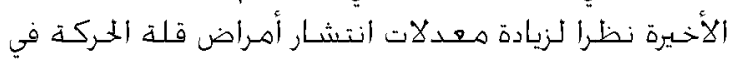

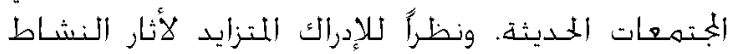

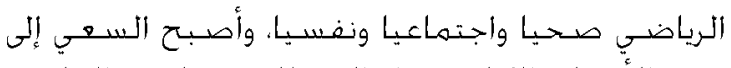

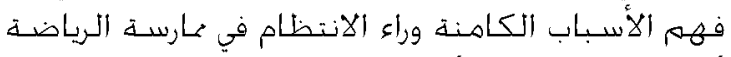

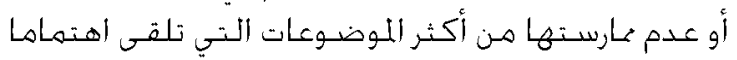

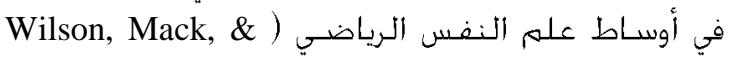

(Grattan, 2008

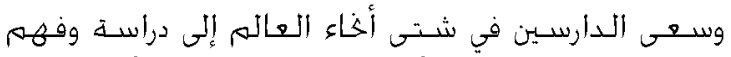

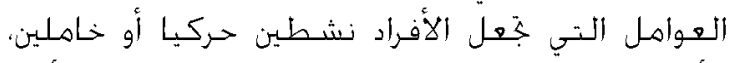

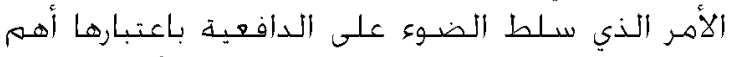

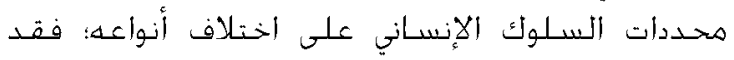

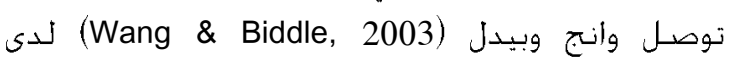

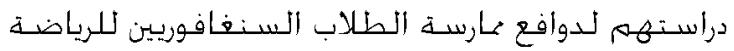


وبالذنب في حالد الخفاض مستوى اللباقة البدنية في المرحلة الجامعية.

أهـاف الدراسـة وأهـمينها

تهـدف هذه الدرأسة الكشف عن دوافع مشـاركة طلاب

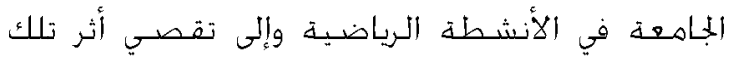

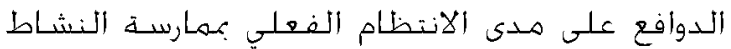

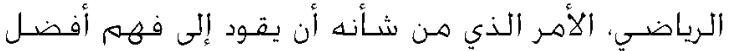

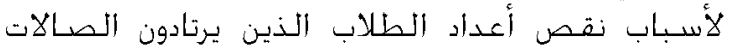

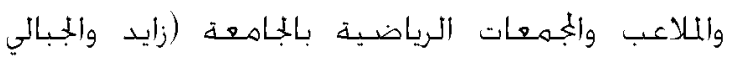

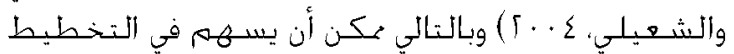

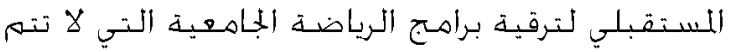

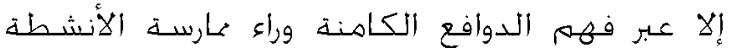
الرياضـيـ (Ingledew \& Markland, 2008).

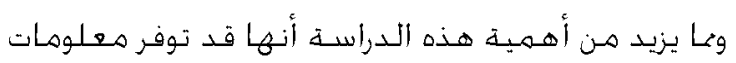

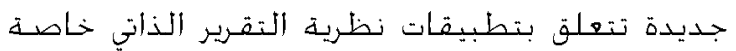

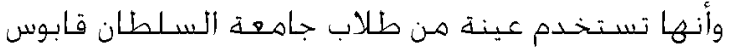

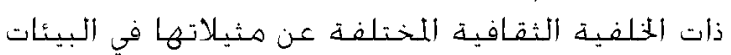

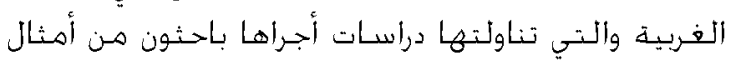

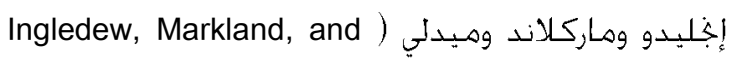
Deci \& Ryan, ) وكذنك ديسي وريان (Medley, 1998 2002) وغيرهـم. أســلة الدراسـة تسـه الدراســة الحالية للإجابة عن الأسئلة التالية: ا. ما دوافع الأنشطة الرياضية لدى طلاب جامعة

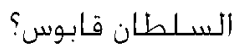

أ. هل حَتلف دوافع الأنشيطة الرياضية لدى طلاب

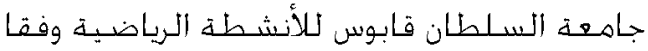

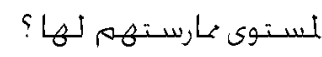

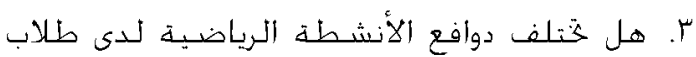

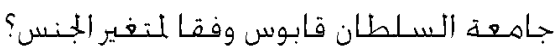

$$
\text { الطريقةـ والإجـراءات }
$$

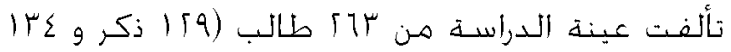

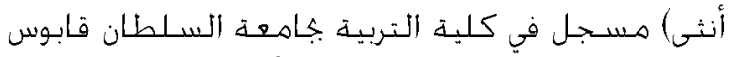

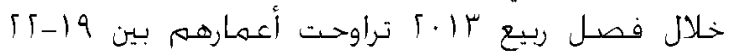

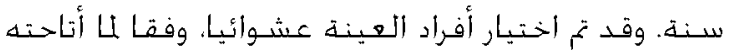

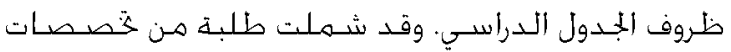

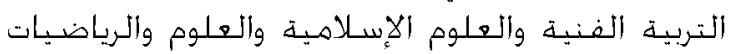

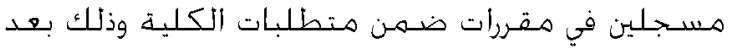

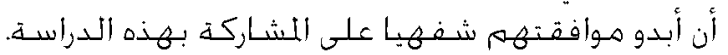

التنظيهم الغير واعي تتنباً بشـة التمـرين بالنسبـة للإناث

فقط.

وأشـار لاو وفان بلجـون وهوجاندأني ( Louw, Vn Biljon, and (Mugandani, 2012

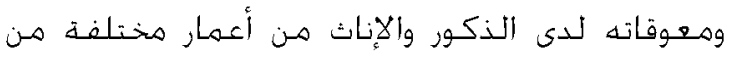

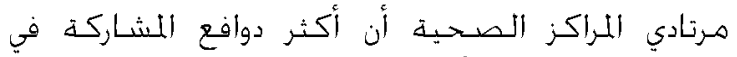

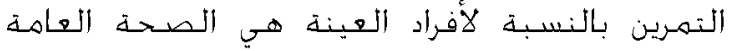

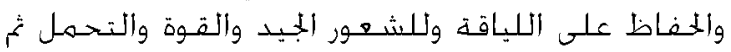

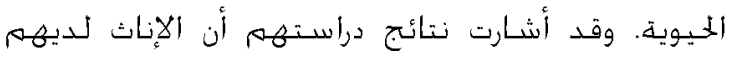

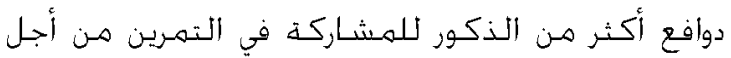
التحكم بالوزن.

وتوصـل كل من تيكيسـيرا وكاراكا وهـاركلاند وسيلفا Teixeira, Carraça, Markland, Silva, and Ryan, ) وريان

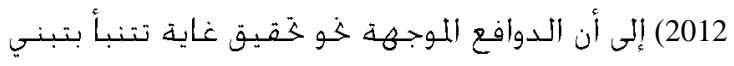

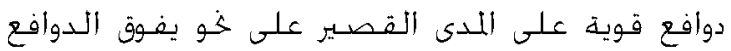
الداخلية، بينما تسـهم الدوافع الداخلية في المئية الستمـرار

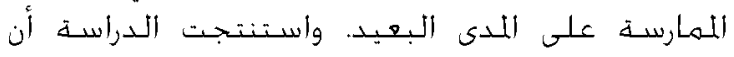

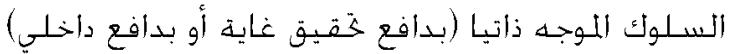

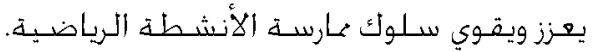

ودرس سـكـولر وبر اندستيتر (Schüler \& Brandstätter, 2013)

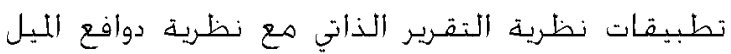
وأثرها في خنسين التنبؤ (Motive Disposition Theory) Flow ) بوصـول الرياضيـين إلى خـبرة الطلاقة (Experience

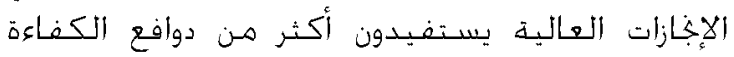

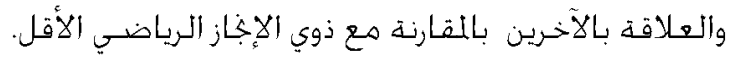

Vartanian, ) وأستنتج كل من فارتينيان ووأرتون وجرين أن أن دوافع المشاركد فين (Wharton, and Green, 2011

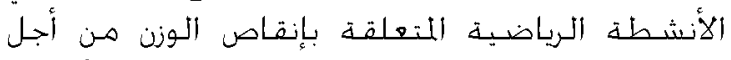

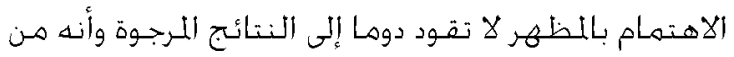

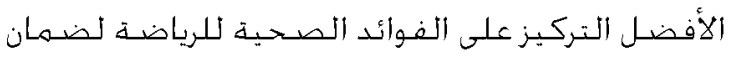

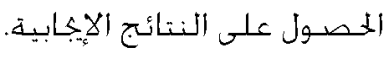

وأخيرا درس كل هن أُورليخ فرينش وكوكس، وبامبس دافهية (Ullrich-French, Cox, \& Bumpus, 2013)

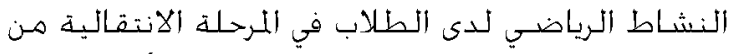

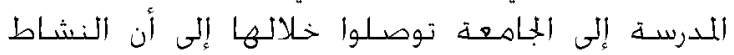

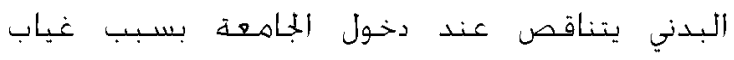

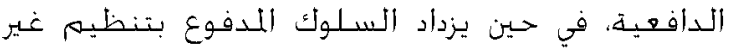

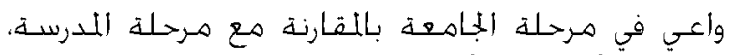

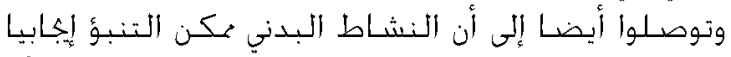

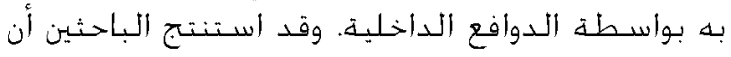

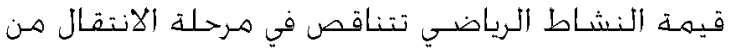

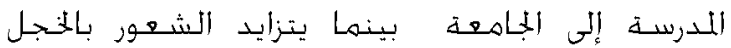


يعملون في جامعة السـلطان قابوس أو في وزارة التربية

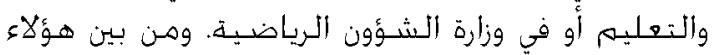

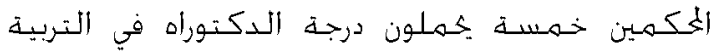

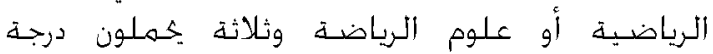

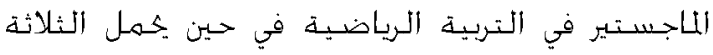

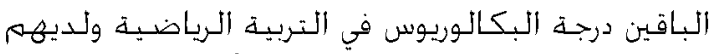

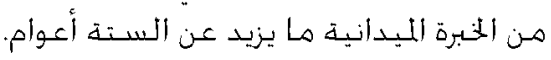

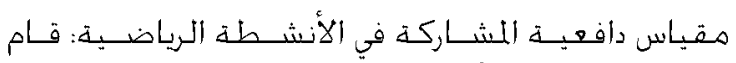

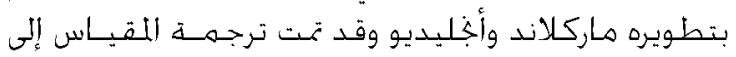

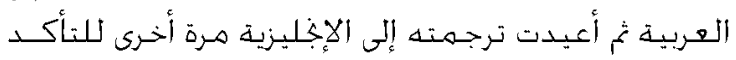

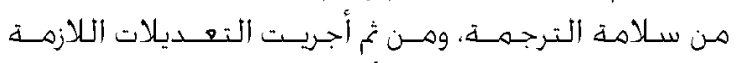

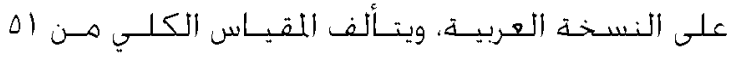

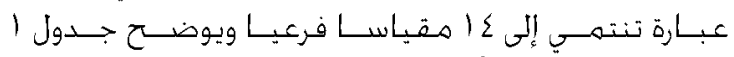

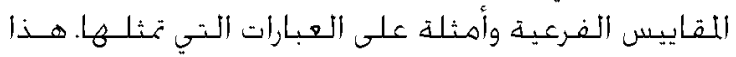

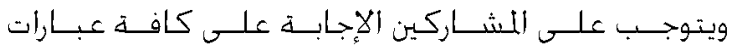

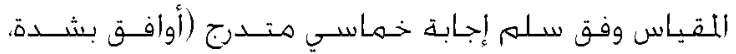

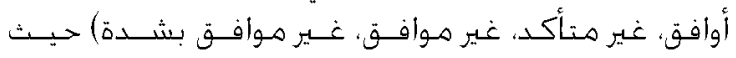

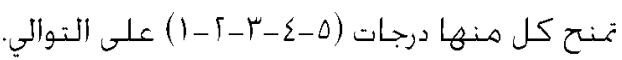

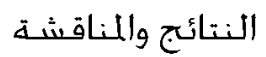

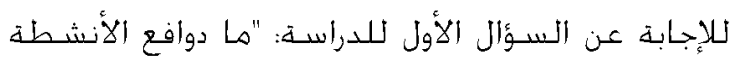

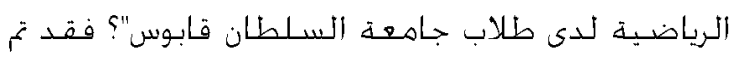

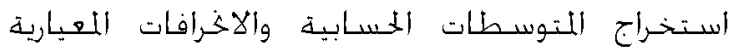

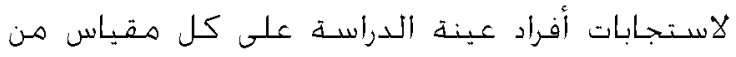

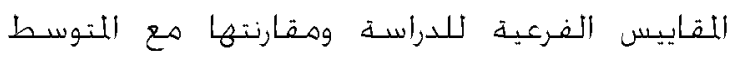

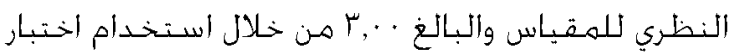

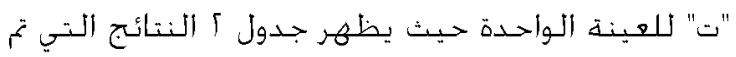

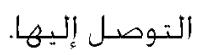

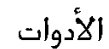

مقياس السلوك الرياضي: وبعتمد على التقرير الذاتي

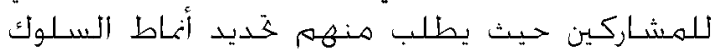

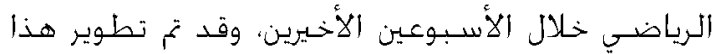
المقباس لأغراض الدراسة ويتألف من ثلاثة أسئلة الأول

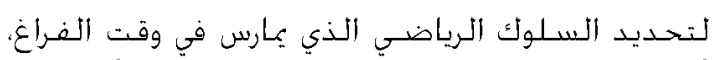

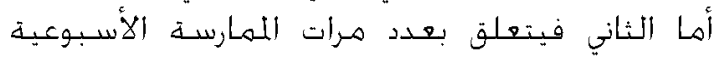

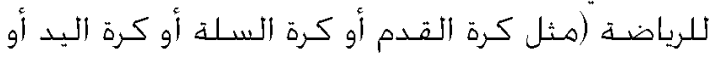

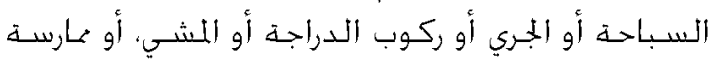

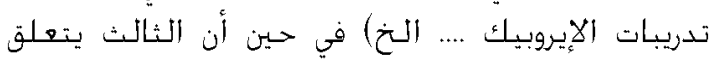

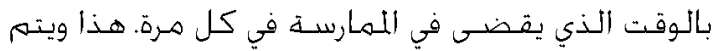

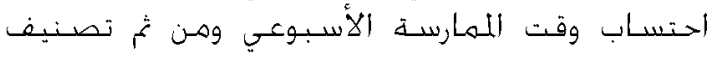

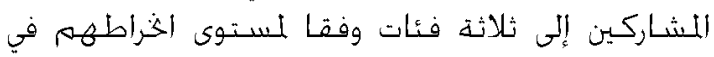

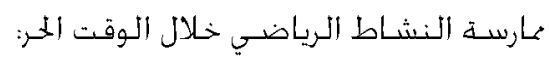

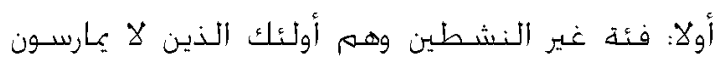

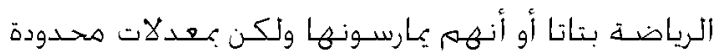
لا تنجاوز · 7 دقيقة أسبوعيا. ثانيا: فئذ معتدلي النشاط وهـم أولئك الذين يمارسـون

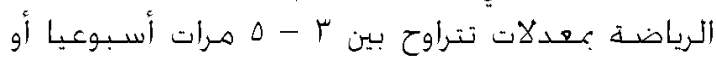

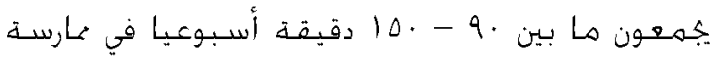
ألرياضذة.

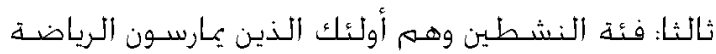

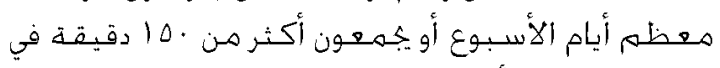
مارسـة الرياضة أسبوعبا.

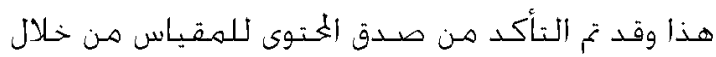

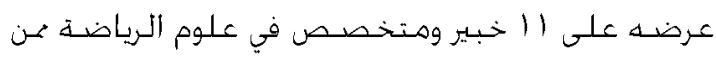

جدول

مقاييس دافعية المشاركة في الأنشطة الرياضية وعدد عباراتها وأمثلة عليها

\begin{tabular}{|c|c|c|c|c|}
\hline أمثلة من العبارات & معامل ألفا & 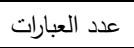 & المقياس الفرعي & 5 \\
\hline أتمرن أو قد أتمرن من أجل أن أتخلص من التوتز & $\cdot, \uparrow$ & $\varepsilon$ & خفض التوتز & 1 \\
\hline أتمرن أو قد أتمرن من اجل أن أعبد شحن طاقتي & 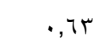 & $r$ & الشعور بالحيوية والنشاط & r \\
\hline أتصرن أو قد أتمرن لأظهر مدى قدرتي البدنية & $\cdot, \vee \vee$ & $\varepsilon$ & الشعور بالتحدي & $\varepsilon$ \\
\hline أتمرن أو قد أتمرن لأحصل على تقدير الآخرين & $\cdot, 10$ & $\varepsilon$ & 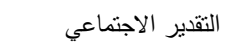 & ○ \\
\hline أتمرن أو قد أتمرن لأنني أحب النتافس مع الآخرين & $\cdot, \wedge \wedge$ & $\varepsilon$ & 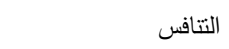 & $v$ \\
\hline أتصرن أو قد أتمرن لأن الطبيب نصحني بذلك & $\cdot, \mathrm{V} r$ & $r$ & 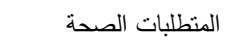 & $\wedge$ \\
\hline أتمرن أو قد أتمرن من أجل الوقاية من الأمراض & $\cdot, \wedge 1$ & $r$ & 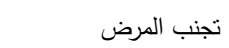 & 9 \\
\hline أتمرن أو قد أتمرن من أجل أن أمتلك الصحة البدنية & $\cdot, v r$ & $r$ & الصحة الايجابية & 1. \\
\hline أتترن أو قد أتمرن من أجل زيادة قوتي العضلية & $\cdot$, , & $\varepsilon$ & القوة والتحمل & r \\
\hline أتمرن أو قد أتمرن من أجل أن أكون رشيقا & $\cdot, 00$ & r & 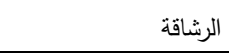 & $1 \varepsilon$ \\
\hline
\end{tabular}

ملاحظة: لم يتم اعتماد مقياس دافعية الرشاقة الفرعي بسبب انخفاض قيمة معامل كرونباخ عن ـ ج, • 
جدول r

المتوسطات الحسابية والانحرافات المعيارية واختبار "ت" لعينة واحدة لكل مقياس من المقاييس الفرعية لدوافع المشاركة في الأنشطة الرياضية (ن = بr بار)

\begin{tabular}{|c|c|c|c|}
\hline قيمة "ت" & الانحراف المعياري & المتوسط الحسابي & المقاييس الفرعية لدوافع المشاركة \\
\hline $19, \cdot \varepsilon$ & $\cdot, 7 \leqslant$ & r,vo & خفض التوتر \\
\hline rq, $\Sigma$ r & $\cdot, 00$ & $\varepsilon, \Gamma \varepsilon$ & الثعور بالحيوية \\
\hline Yr,VT & $\cdot, \mathrm{TV}$ & ५, q & الثعور بالمتعة \\
\hline $19, \wedge r$ & $\cdot, \uparrow \wedge$ & r ( & الثعور بالتحدي \\
\hline$+1, \vee \wedge \wedge-$ & $1, \cdot 1$ & $r, \wedge \wedge$ & التقدير الاجتماعي \\
\hline$Y V, \Sigma T$ & ז ג, & $r, q$. & الانتماء \\
\hline$\wedge, \varepsilon \varepsilon$ & $1, \cdot r$ & $r, 0 \leqslant$ & التنتافس \\
\hline $1 T, \varepsilon$. & $\cdot, 9$ & $r, v \varepsilon$ & المطالب الصحية \\
\hline ro, rq & 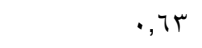 & $\varepsilon, \Gamma \wedge$ & تجنب المرض \\
\hline$r_{\Lambda}, 1 \leq$ & $\cdot, 01$ & $\varepsilon, r V$ & الصحة الايجابية \\
\hline Ir,rV & $\cdot, 90$ & $r, v r$ & التحكم بالوزن \\
\hline $10, \leqslant Y$ & $\cdot$, Ar & r,v^ & الاهتمام بالمظهر \\
\hline 9,17 & $1, \cdot r$ & $r, 0 \wedge$ & القوة والتحمل \\
\hline
\end{tabular}

+ غير دال إحصائياً عند مستوى 0.,.•، باقي قيم ت كلها دالة عذد مستوى ا.,.

(Kilpatrick et al., 2005) إلبه كل من كلباتريك ورفاقه

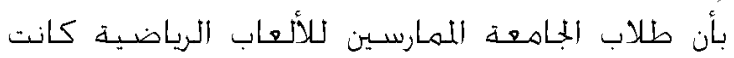

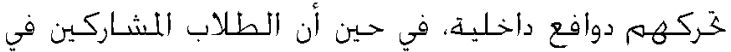

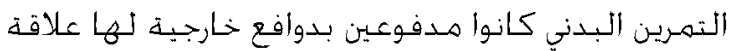

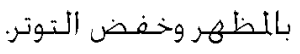

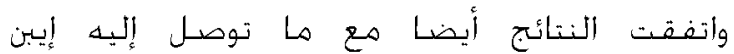
وبريكينسيكي (Ebben \& Brudzynski, 2008) من أن دوافع مشاركة طلاب الجامعة بالأنشطة الرياضـية

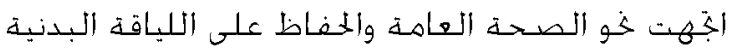

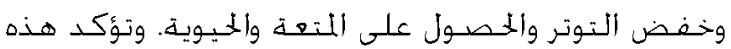

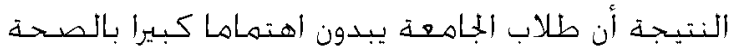
وباللياقة البدنية، وفي نفس الوفت الذي يسعون فيه

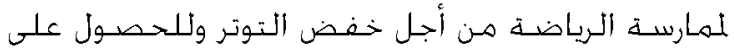

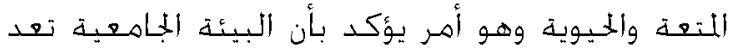

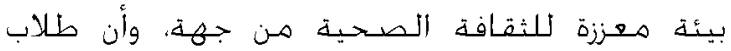

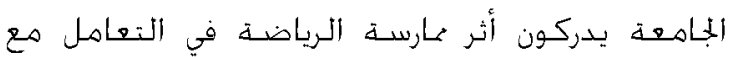

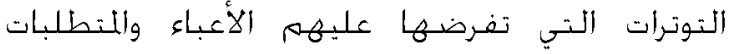
الدراسـية، من جهية ثانية.

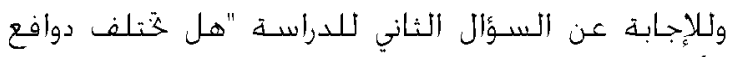

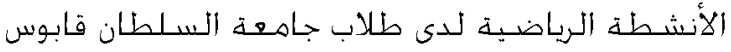

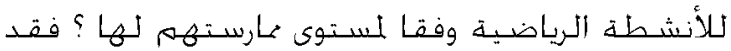

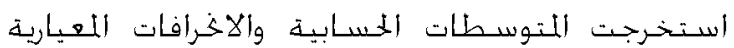

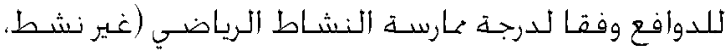
هعتدل النشاط، نشـط)، وجـدول بـ يبين ذلك.

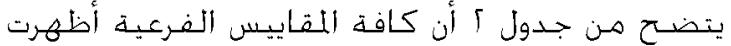

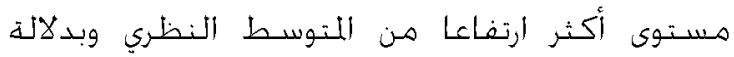

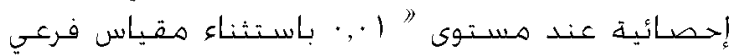

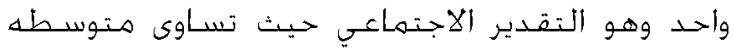

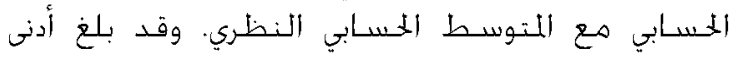

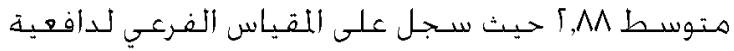

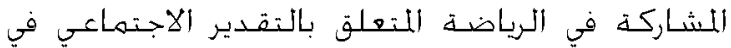

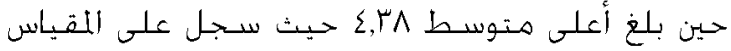

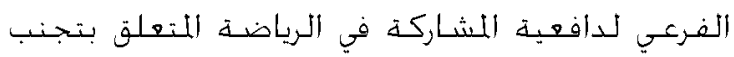
المرض.

وتشير هذه النتبجه أن أكثر دوافع ميارسـة الأنشطة

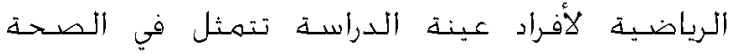

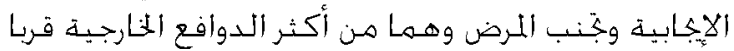

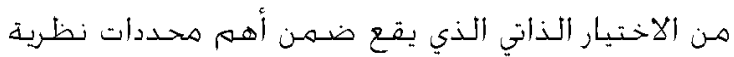

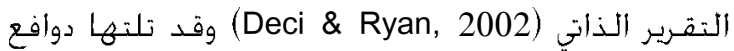

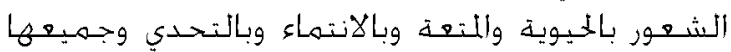

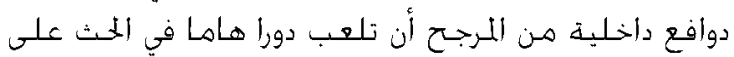
القيام بالسـلوك الرياضي (Kilpatrick et al., 2005). ويشسير جدول ا أيضـا إلى أن كل هن دافع الاهتمام

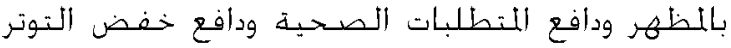

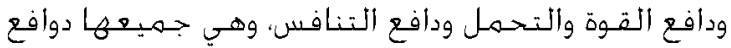

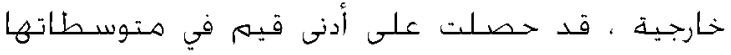

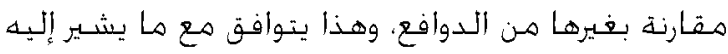

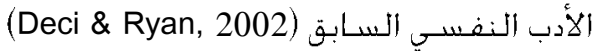
ويلاحذظ أن لدى طلاب الجامعة مـزيكا من الدوافع الداخلية

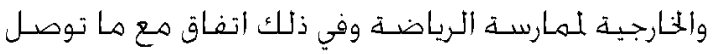


جدول باف المشارs

\begin{tabular}{|c|c|c|c|c|c|c|}
\hline \multicolumn{2}{|c|}{ نشط (ن=V } & \multicolumn{2}{|c|}{ معتدل النشاط (ن= (NV) } & \multicolumn{2}{|c|}{ غير نشط (ن= Y Y I ) } & \multirow[b]{2}{*}{ المقاييس الفرعية لدوافع المشاركة } \\
\hline الانحراف & المتوسط الحسابي & الانحراف & المتوسط الحسابي & الانحراف & المتوسط الحسابي & \\
\hline$\cdot, 7 \varepsilon$ & $\varepsilon, r V$ & $\cdot, \leqslant 9$ & $r, 7 \Lambda$ & זוז, 年 & $r, 7)$ & خفض التوتز \\
\hline • & $\varepsilon, \gamma$ & $\cdot, \varepsilon \varepsilon$ & $\varepsilon, r \uparrow$ & $\cdot, 7$ & $\varepsilon, r_{\text {. }}$ & الشعور بالحيوية \\
\hline$\cdot$, ov & $\varepsilon, \varepsilon$ & $\cdot, 70$ & $r, 94$ & $\cdot, 70$ & $r, r v$ & الثعور بالمتعة \\
\hline$\cdot, 7$ & $\varepsilon, r)$ & $\cdot, 0$ & $\varepsilon, \cdot r$ & $\cdot$, TV & r,or & الثعور بالتحدي \\
\hline $1, \cdot 1$ & r,or & $\cdot, \wedge \mathrm{V}$ & $r, .9$ & $1, \cdot v$ & $r, 0$. & التقدير الاجتماعي \\
\hline$\cdot, 7$ & $\varepsilon, r$. & $\cdot$, vo & $r, v \wedge$ & $\cdot, 91$ & r,یr & الانتماء \\
\hline$\cdot, \wedge \varepsilon$ & $\varepsilon, r r$ & $\cdot, 9$. & r, & $1, \cdot 1$ & $r, 19$ & 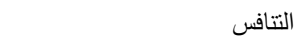 \\
\hline$\cdot, \mathrm{V} \varepsilon$ & $r, \wedge q$ & $\cdot, 10$ & $r, \wedge$. & $\cdot, 9 \vee$ & $r, 70$ & المطالب الصحية \\
\hline$\cdot, \leqslant 9$ & $\varepsilon, 00$ & $\cdot, 7)$ & $\varepsilon, r$. & $\cdot, 79$ & $\varepsilon, \Gamma \wedge$ & تجنب المرض \\
\hline$\cdot, \wedge r$ & $\varepsilon, r 4$ & $\cdot, \leqslant 0$ & $\varepsilon, r V$ & $\cdot$, ov & $\varepsilon, \Gamma \wedge$ & الصحة الايجابية \\
\hline$\cdot, \wedge \cdot$ & r,vi & $1, .0$ & $r, \pi 4$ & $\cdot, 94$ & $r, v \varepsilon$ & التحكم بالوزن \\
\hline$\cdot, 91$ & $\varepsilon,$. & $\cdot, \vee Y$ & $r, V r$ & $\cdot, \mathrm{YA}$ & $r, v r$ & الاهتمام بالمظهر \\
\hline$\cdot, 7 \varepsilon$ & $\varepsilon, \varepsilon 1$ & $\cdot, 9 \vee$ & $\Gamma, \wedge \varepsilon$ & $\cdot, 9 \leqslant$ & $r, 11$ & القوة والتحمل \\
\hline
\end{tabular}

نتائج تحليل التباين المتعدد لأثر متغير مستوى ممارسة النثاط الرياضي في دوافع المشاركة

\begin{tabular}{|c|c|c|c|c|c|c|}
\hline مربع إيتا & قيمة "ف" & متوسط المربعات & درجات الحرية & مجموع المربعات & مقاييس دافعية المشاركة & مصدر التباين \\
\hline., $1 \leq V$ & $* *$ * Y r T & $\mathrm{V}, \wedge)$ & $r$ & $(0,7)$ & خفض التوتز & \\
\hline., $11 r$ & $* * 17, r$ & $\{, \varepsilon \uparrow$ & r & $\wedge, 94$ & الشعور بالحيوية & \\
\hline$\cdot, 111$ & $* * \mid V, \leq 7$ & $V, \cdot \varepsilon$ & r & $1 \leq, .9$ & الشعور بالمتعة & \\
\hline$\cdot, r \cdot q$ & $* * r \leq, \varepsilon r$ & $M, T \leq$ & r & ro, rV & الشعور بالتحدي & \\
\hline • & $* * r \cdot, 09$ & $r \cdot, l \cdot$ & r & $\leqslant 1,99$ & التقدير الاجتماعي & \\
\hline$\cdot$, . or & $* v, 1$ & $\varepsilon, 79$ & r & $৭, \Gamma \wedge$ & 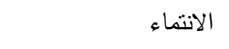 & مستوى ممارسة الرياضة \\
\hline ת & $* *$ ro, rq & $r Y, V \leq$ & r & $\leqslant 0, \leqslant \wedge$ & 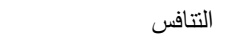 & \\
\hline$\cdot, \cdot{ }^{\prime}$ & אדר, & I,r. & r & $r, \tau$. & المطالب الصحية & \\
\hline$\cdot, .19$ & $r, \varepsilon Y$ & $\cdot, 9 \vee$ & r & $1,9 \leq$ & تجنب المرض & \\
\hline$\cdot, \cdots$ & $\cdot, \cdot \leq \varepsilon$ & $\cdot, \cdot 1$ & r & $\cdot, \cdot r$ & الصحة الايجابية & \\
\hline$\cdot, \cdots r$ & • & $\cdot, r q$ & r & $\cdot, 0 \wedge$ & التحكم بالوزن & \\
\hline$\cdot, 17$ & $r, \cdot T$ & 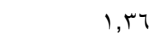 & r & $r, V r$ & الاهتمام بالمظهر & \\
\hline • & $* * \varepsilon \cdot, \uparrow \wedge$ & O & r & $74, \leqslant 9$ & القوة والتحمل & \\
\hline
\end{tabular}

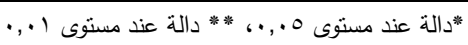

ملاحظة: ثم استبعاد مصدر الخطأ لتوفير الصفحات.

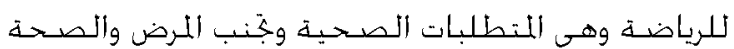

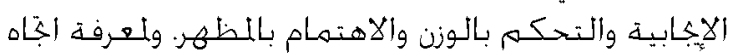

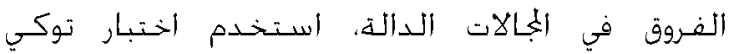

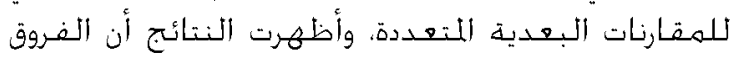

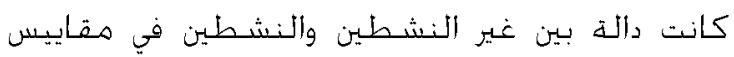

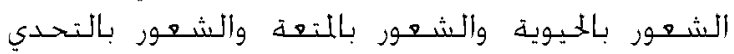

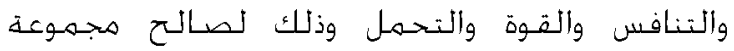

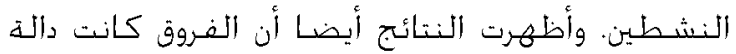

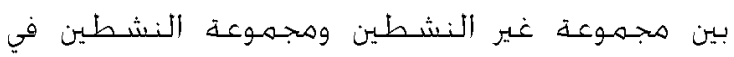

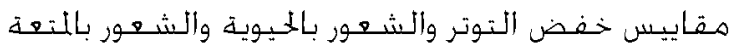

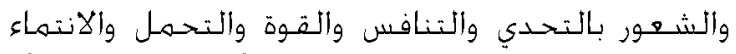

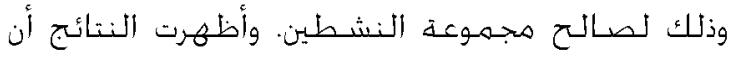

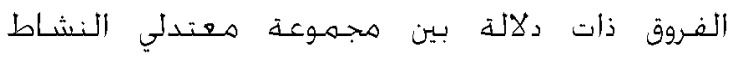

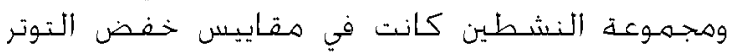

وفـ أستخـدم بعد ذلك ختليل التباين المتعدد للتحقق هن

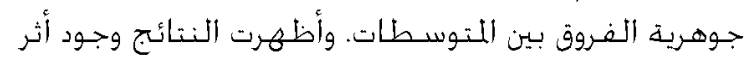

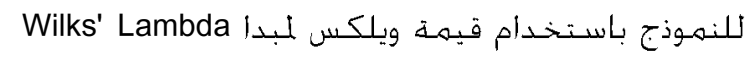

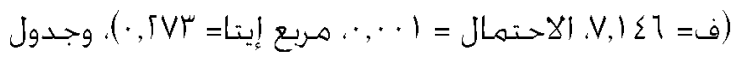
ع يبين خلاصـة نتائج خحليل التباين. يبين جدول ع وجود تأثير ذي دلالة إحصائية لتنغير مسنوى

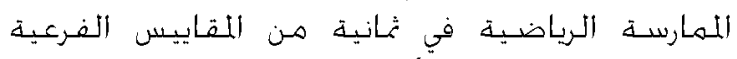

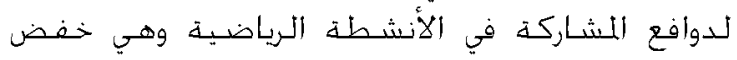

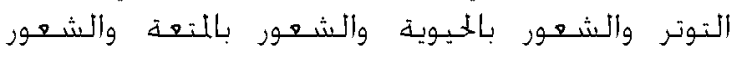

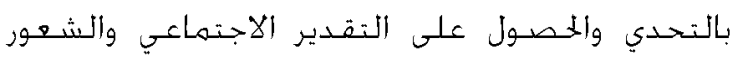

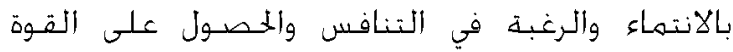

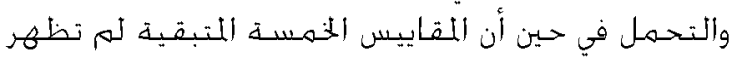

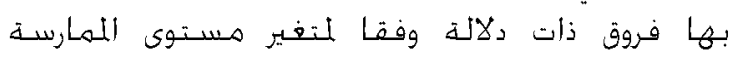


وأظههرت ألنتائج أيضا وجهود فروق ذات دلالة إحصائية بين

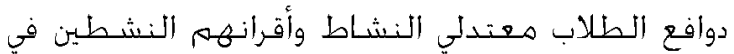

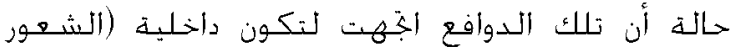

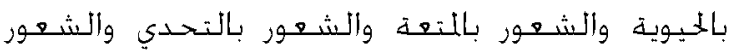

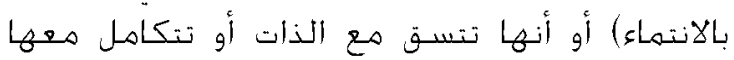

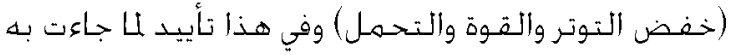

نظرية التحديد الذاتي (Deci \& Ryan, 2002).

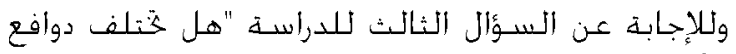

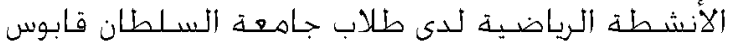

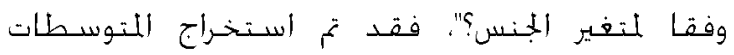

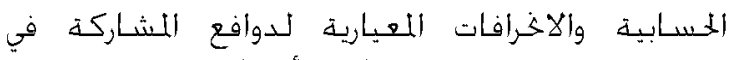

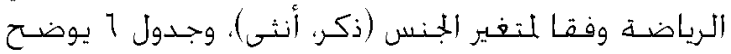

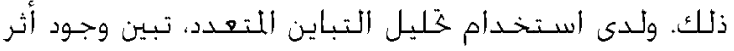

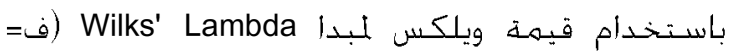
(Y,q)

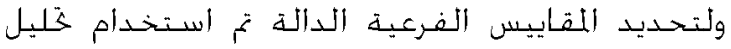
التبابن الأحادي، وجدول V يبين خلاصدة نتائج خليل

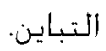

والشعور بالحبوية والشعور بالمتعة والشعور بالتحدي والشهي

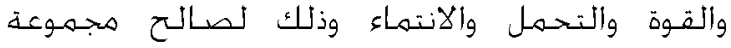

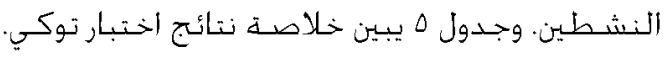

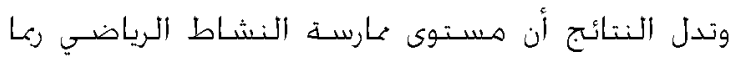
تتأثر بدوافع المشاركة في الأنشطة الرياضبذ وخاصنة

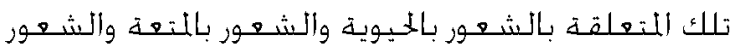

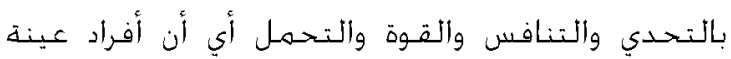

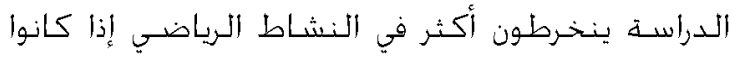

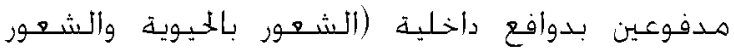

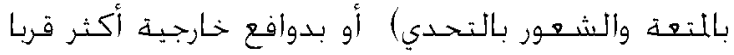
من الاختيار المحر (التنافس والقوة والتحملـ).

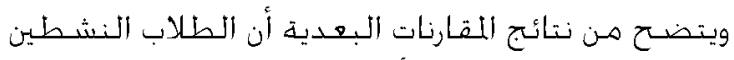

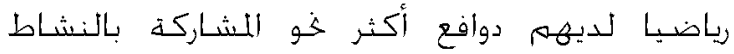

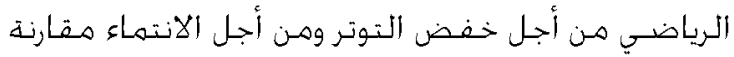

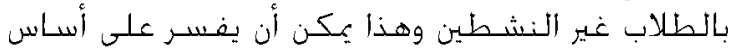

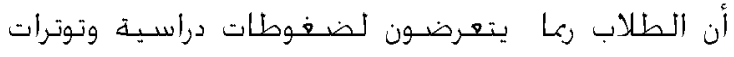

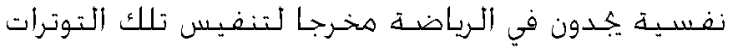

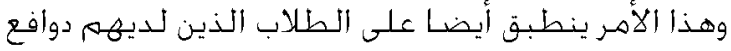

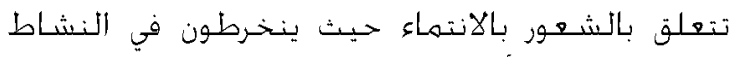
الرياضب بشكل أكبر.

جدول

نتائج اختبار توكي للمقارنات البعدية المتعددة في المقاييس الفرعية الدالة

\begin{tabular}{|c|c|c|c|}
\hline الدلالة الإحصائية & فروق المتوسطات & المقارنات الثنائية & مقاييس دافعية المشاركة \\
\hline • זNT & $\cdot, \cdot \vee-$ & غير نشط × معتدل النشاط & خفض التوتر \\
\hline$\cdot, \cdots 1$ & $\cdot, 74-$ & غير نشط × نشط & \\
\hline$\cdot, \ldots 1$ & $\cdot, 09-$ & معتدل النشاط × نشط & \\
\hline$\cdot, . r^{\prime}$ & $\cdot, 17-$ & غير نشط × معتدل النشاط & الثعور بالحيوية \\
\hline$\cdot, \ldots 1$ & $\cdot, 01-$ & غير نشط × نشط & \\
\hline$\cdot, \cdots$ & $\cdot$, ro- & معتدل النشاط × نشط & \\
\hline$\cdot, \cdot$ ץA & $\cdot, 19-$ & غير نشط × معتدل النشاط & الشعور بالمتعة \\
\hline$\cdot, \cdots 1$ & $\cdot, 7 \leqslant-$ & غير نشط × نشط & \\
\hline$\cdot, \cdots$ & $\cdot, \leq \leqslant-$ & معتدل النشاط × نشط & \\
\hline$\cdot, \cdots 1$ & $\cdot, \leqslant 9-$ & غير نشط × معتدل النشاط & الشعور بالتحدي \\
\hline$\cdot, \cdots 1$ & $\cdot, \mathrm{\vee} \wedge-$ & غير نشط × نشط & \\
\hline$\cdot, \cdots 9$ & $\cdot, r_{9-}$ & معتدل النشاط × نشط & \\
\hline$\cdot,, \cdots$ & $\cdot, 01-$ & غير نشط × معتدل النشاط & النقير الاجتماعي \\
\hline$\cdot, \cdots 1$ & $1, \cdot r-$ & غير نشط × نشط & \\
\hline$\cdot, .17$ & $\cdot, \leq \leqslant-$ & معتدل النشاط × نشط & \\
\hline$\cdot, \vee \cdot \varepsilon$ & $\cdot, \cdot \varepsilon$ & غير نشط × معتدل النشاط & الالتماء \\
\hline$\cdot, \cdots$, & $\cdot, \varepsilon \vee-$ & غير نشط × نشط & \\
\hline$\cdot, \cdots 1$ & $\cdot$, OY- & معتدل النشاط × نشط & \\
\hline$\cdot, \ldots 1$ & •, $\varepsilon T_{-}$ & غير نشط × معتدل النشاط & التنافس \\
\hline$\cdot,, \cdots$ & $1,1 \leqslant-$ & غير نشط × نشط & \\
\hline$\cdot, \cdots 1$ & $\cdot, \vee>-$ & متدل النشاط × نشط & \\
\hline$\cdot, \cdots 1$ & $\cdot, \mathrm{VY}-$ & غير نشط × معتدل النشاط & القوة والتحمل \\
\hline$\cdot, \cdots 1$ & $1, r q-$ & غير نشط × نشط & \\
\hline$\cdot, \ldots 1$ & $\cdot, 0 \mathrm{~V}-$ & معتدل النشاط × نشط & \\
\hline
\end{tabular}


جدول 1

\begin{tabular}{|c|c|c|c|c|}
\hline \multicolumn{2}{|c|}{ إناث (ن= \& ا (1) } & \multicolumn{2}{|c|}{ ذكور (ن= qr או) } & \multirow{2}{*}{ الدقاييس الفرعية لدوافع المشاركة } \\
\hline الانحراف المعياري & المتوسط الحسابي & الانحراف المعياري & المتوسط الحسابي & \\
\hline$\cdot, 70$ & $r, o v$ &., $0 \mathrm{~V}$ & $r, q r$ & خفض التوتز \\
\hline., 09 & $\varepsilon, r \varepsilon$ & $\cdot, \leqslant 9$ & $\leq, \leq 7$ & الشعور بالحيوية \\
\hline$\cdot, 74$ & $r, \wedge)$ & 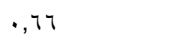 & $\varepsilon, \cdot \wedge$ & الثعور بالمتعة \\
\hline$\cdot, 79$ & $r, 09$ & $\cdot, 0 \mathrm{~V}$ & $\varepsilon, \bullet$ & الشعور بالتحدي \\
\hline r & $r, r v$ & $\cdot, 9 \vee$ & $r, \varepsilon 1$ & التقدير الاجتماعي \\
\hline$\cdot, \wedge 4$ & r,vo & $\cdot, \vee \wedge$ & $\varepsilon, \cdot \varepsilon$ & الانتماء \\
\hline $1, \cdot r$ & $r, \cdot \Lambda$ & $\cdot, \vee \vee$ & $\varepsilon, \cdot 1$ & التنافس \\
\hline$\cdot, 9 \leqslant$ & r,Vr & $\cdot, \wedge 4$ & $r, v ч$ & المطالب الصحية \\
\hline$\cdot$, TV & $\varepsilon, \varepsilon r$ &., 09 & 每 & تجنب المرض \\
\hline r & $\varepsilon, \varepsilon 1$ &., $0 \leqslant$ & $\varepsilon, \Gamma \varepsilon$ & الصحة الايجابية \\
\hline$\cdot, 91$ & $r, \wedge \wedge$ & $\cdot, 97$ & $r, \infty 0$ & التحكم بالوزن \\
\hline$\cdot, \vee \vee$ & $r, \wedge \Lambda$ & $\cdot, \wedge \Lambda$ & $r, T V$ & الاهتمام بالمظهر \\
\hline$\cdot, \lambda \mathrm{V}$ & $r, q r$ & $\cdot, T V$ & $\varepsilon, \Upsilon \wedge$ & القوة والتحمل \\
\hline
\end{tabular}

نتائج تحليل التباين المتعدد لأثر متغير النوع في دوافع المشاركة بالأنثطة الرياضية

\begin{tabular}{|c|c|c|c|c|c|c|}
\hline مربع ايتا & قيمة "ف" & متوسط المربعات & درجات الحرية & مجموع المربعات & مقاييس دافعية المشاركة & مصدر التباين \\
\hline$\cdot, \cdot 11$ & $* * r, q$. & 1,01 & 1 & 1,01 & خفض التوتر & \multirow{13}{*}{ النوع الاجتماعي } \\
\hline$\cdot, \cdot \leqslant$ & $* * 1 \cdot, 10$ & $r, 19$ & 1 & $r, 19$ & الثعور بالحيوية & \\
\hline.,.$r q$ & $* * 1 \cdot, \pi r$ & $\varepsilon, 70$ & 1 & $\varepsilon, 70$ & الشعور بالمتعة & \\
\hline o & $* * \varepsilon \cdot, \wedge \wedge$ & $17, r \varepsilon$ & 1 & $17, r \leqslant$ & الشعور بالتحدي & \\
\hline D & $* * \wedge \vee, T \leq$ & VI, Ir & 1 & VI, & التقدير الاجتماعي & \\
\hline ,.r. & $* * \wedge, 19$ & 0,01 & 1 & 0,01 & الانتماء & \\
\hline$\cdot, r \cdot V$ & $* * \uparrow \wedge, r q$ & $O V, V y$ & 1 & ov,VY & التنافس & \\
\hline.,$\ldots 1$ & $\cdot, 1 \leq 0$ & $\cdot, I r$ & 1 & ., Ir & المطالب الصحية & \\
\hline$\cdot, \ldots$ & $1, \wedge \varepsilon$ & $\cdot, 09$ & 1 & $\cdot, 09$ & تجنب المرض & \\
\hline,$\ldots \varepsilon$ & . & . & 1 & 酒 & الصحة الايجابية & \\
\hline$\cdot, \cdot r)$ & $* * \wedge, r r$ & $V, Y_{O}$ & 1 & $V, r_{O}$ & التحكم بالوزن & \\
\hline$\cdot, \cdot 11$ & $* \varepsilon, A r$ & $r, i v$ & 1 & $r, I V$ & الاهتمام بالمظهر & \\
\hline \multirow[t]{14}{*}{., $\varepsilon r_{0}$} & $* * r \cdot 1, \cdot \wedge$ & $|r|, \varepsilon r$ & 1 & $|r|, \varepsilon r$ & القوة والتحمل & \\
\hline & & $\cdot, \Gamma \wedge$ & Y4I & $\cdot, \wedge 9 \vee$ & خفض التوتز & \multirow{13}{*}{ الخطأ } \\
\hline & & $\cdot, r q$ & rri & 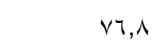 & الشعور بالحيوية & \\
\hline & & $\cdot, \varepsilon \varepsilon$ & rri & $11 \leqslant, r$ & الشعور بالمنعة & \\
\hline & & $\cdot, \varepsilon$. & rTl & $1 \cdot \varepsilon, \varepsilon$ & الشعور بالتحدي & \\
\hline & & $\cdot, 9$ & ry & EMr, & التقدير الاجتماعي & \\
\hline & & $\cdot$, TV & rq & $1 v 0,00$ & الانتماء & \\
\hline & & $\cdot, 10$ & ry & rr.,OV & التنافس & \\
\hline & & $\cdot, \wedge 1$ & rn & $r 1 \cdot, \varepsilon V$ & المطالب الصحية & \\
\hline & & $\cdot, \varepsilon \cdot$ & rYl & $1 \cdot \varepsilon, 11$ & تجنب المرض & \\
\hline & & • & rTI & $\wedge \Lambda, q Y$ & الصحة الايجابية & \\
\hline & & $\cdot, \wedge V$ & rul & $r r V, r q$ & التحكم بالوزن & \\
\hline & & . & rul & $|v|, v r$ & الاهتمام بالمظهر & \\
\hline & & $\cdot, \pi$ & rYl & $10 V, 7$ & القوة والتحمل & \\
\hline
\end{tabular}

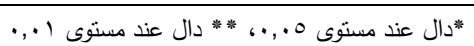

والشهور بالتحدى والخهـول على التقدير الاجتماعي

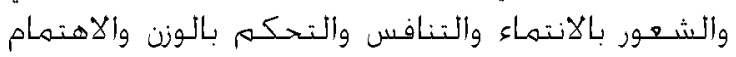

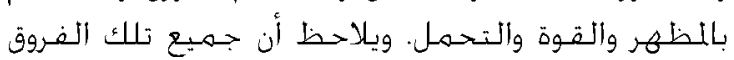

يشير جدول V إلى وجود فروق ذات دلالة بين الذكور والإناث

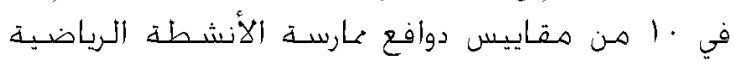

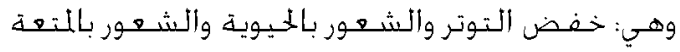




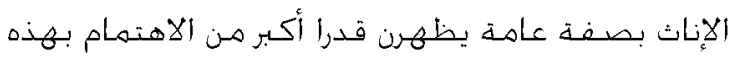

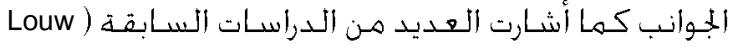

et al., 2012; Kilpatrick et al., 2005; Vartanian et al.,

(2011

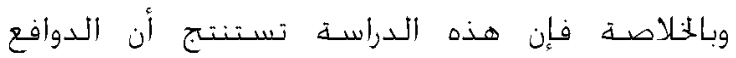

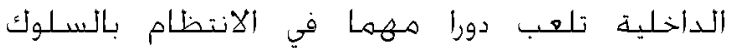

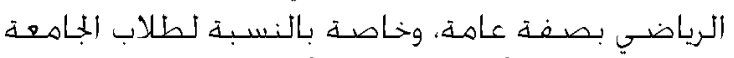

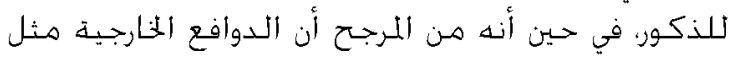

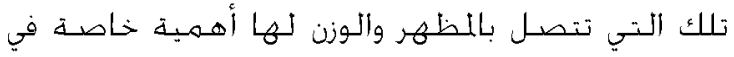
توفع سـلوك الطلالبات الإناث الرياضي وفي ذلك تهضيد

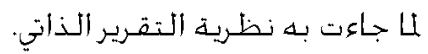

$$
\text { المراجـ }
$$

زأيد، كاشف والجبالي، هصـفي، وألشهيلي، علي

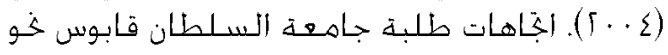

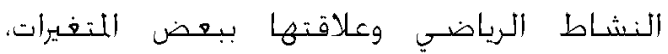

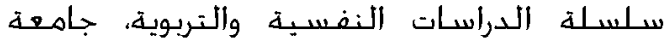
السلطان قابوس، العدد ^ه

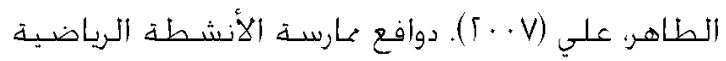

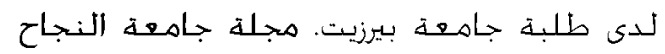

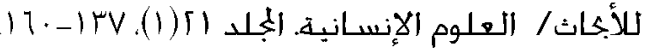

Cavlo, T. G., Cervelló, E., Jiménez, R., Iglesias, D., \& Murcia, J. A. M. (2010). Using selfdetermination theory to explain sport persistence and dropout in adolescent athletes. The Spanish Journal of Psychology, 13(2), 677-684.

Deci, E. L., \& Ryan, R. (2002). Handbook of selfdetermination research. NY. Rochester: University of Rochester Press.

Duncan, L., Hall, C., Wilson, P. Jenny, O. (2010). Exercise motivation: A cross-sectional analysis examining its relationships with frequency, intensity, and duration of exercise. International Journal of Behavioral Nutrition and Physical Activity.7 doi: 10.1186/1479-5868-7-7.

Ebben, W., \& Brudzynski, L. (2008). Motivations and barriers to exercise among college students. Journal of Exercise Physiology Online, 11(5), 1-11.

Ingledew, D. K., Markland, D. and Ferguson, E. (2009), Three levels of exercise motivation. Applied Psychology: Health and Well-Being,
كانت لصسالح الذكور باسـتثناء التحكم بالوزن والاهتمام

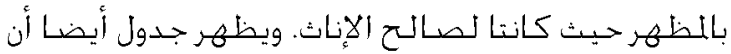

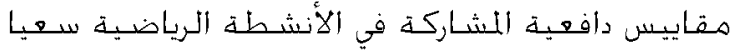

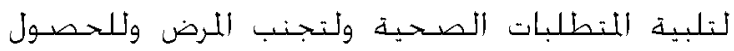

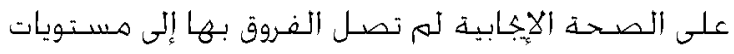

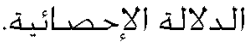

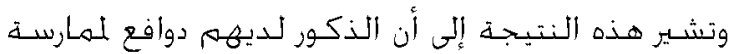

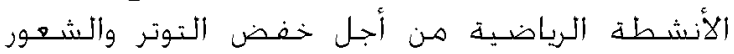

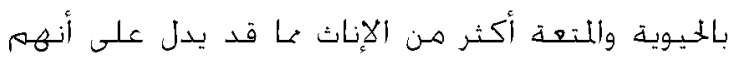

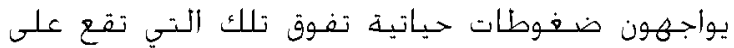

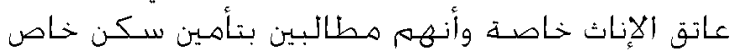

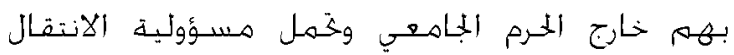

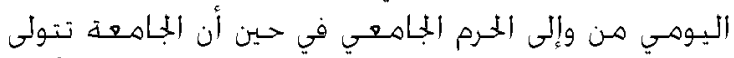

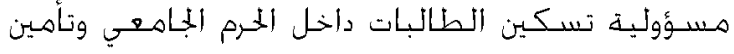

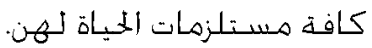

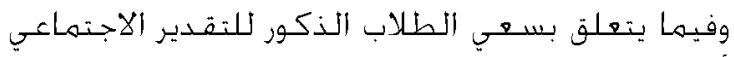

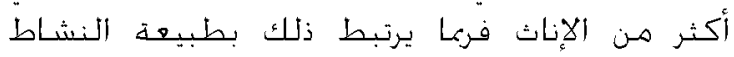

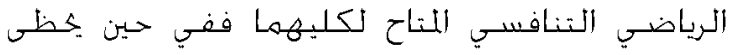

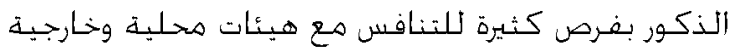

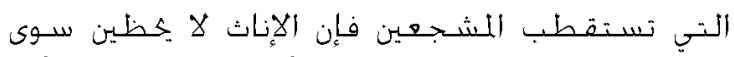

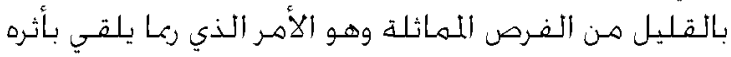

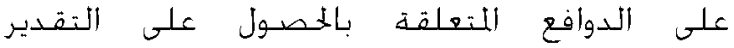

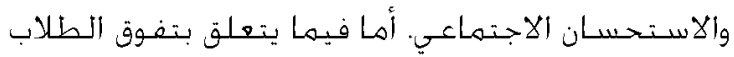

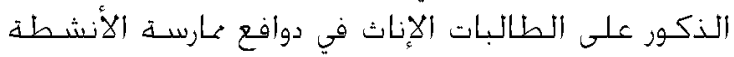

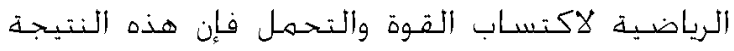

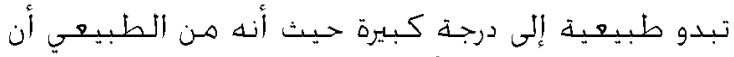

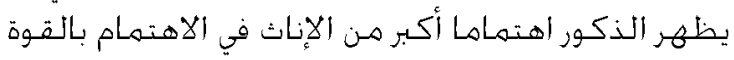

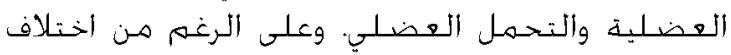
النتائج التي توصلت إليد هذه الدراسـد وخاصـة فيها

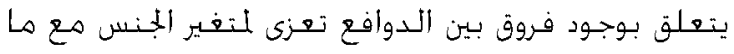

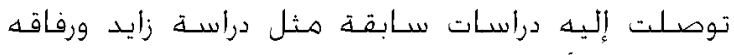

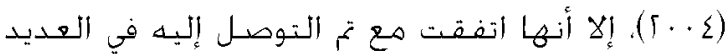

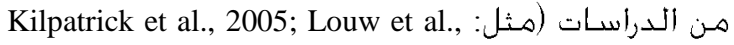

.). (2012

أَها فيما يتهلق بتفوق الطلاب على الطظالبات في دوافع

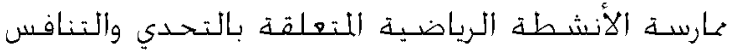

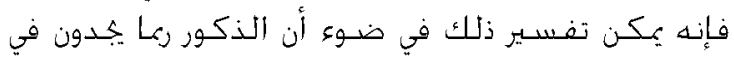

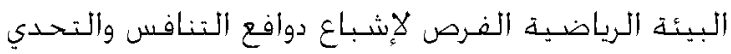

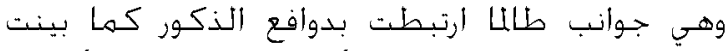

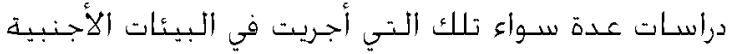

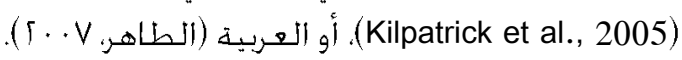
وأخيراً فإن وجود فروق ذات دلالة إحصائية بين دوافع

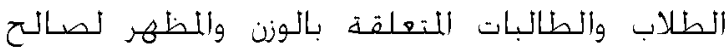
الطالبات ينظر إليه باعتباره طبيهيا ومتوقها حيث أن والن لمهن 
1, 336-355. doi: 10.1111/j.1758-0854.2009.01015.

Ingledew, D., \& Markland, D. (2008). The role of motives in exercise participation. Psychology of Health, 23(7): 807-828).

Kilpatrick, M., Hebert, E., and Bartholomew, J. (2005). College Students' Motivation for Physical Activity: Differentiating Men's and Women's Motives for Sport Participation and Exercise. Journal of American College Health. 54(2), 87-94.

Landry, J., \& Solmon, M. (2004). African American women's self-determination across the stages of change for exercise. Journal of Sport and Exercise Psychology, 26, 457-469.

Louw, A. J., Vn Biljon, A., and Mugandani, S. C. (2012). Exercise motivation and barriers among men and women of different age groups. African Journal for Physical Education, Recreation, and Dance, 18(4), 759-768.

Murcia, J., Roman, M., Galindo, C., Alonso, N., \& Gonzalez-Cutre, D. (2008). Peers' influence on exercise enjoyment: A self-determination theory approach. Journal of Sports Science and Medicine, 7, 23-31.

Ryan, R., Williams, G., Patrick, \& Deci, E. (2009). Self-determination theory and physical activity: The dynamics of motivation in development and wellness. Hellenic Journal of Psychology, 6, 107 - 124.

Ryan, R.M. and Deci, E.L. (2000). Selfdetermination theory and the facilitation of intrinsic motivation, social development, and well-being. American Psychologist, 55, 6878.

Schüler, J. and Brandstätter, V. (2013). How basic need satisfaction and dispositional motives interact in predicting flow experience in sport. Journal of Applied Social Psychology, 43, 687-705. doi: 10.1111/j.15591816.2013.01045.

Teixeira, P., Carraça, E., Markland, D., Silva, M., and Ryan, R. (2012) Exercise, physical activity, and self-determination theory: A systematic review. International Journal of Behavioral Nutrition and Physical Activity, 9, 13.

Tsai, Y., Kunter, M., Lüdtke, O., Trautwein, U., \& Ryan, R. M. (2008). What makes lessons interesting? The role of situational and individual factors in three school subjects. Journal of Educational Psychology, 100, 460472. DOI: $10.1037 / 0022-0663.100 .2 .460$
Ullrich-French, S., Cox, A., \& Bumpus, M. (2013). Physical activity motivation and behavior across the transition to university. Sport, Exercise, and Performance Psychology, 2, 90101. doi:10.1037/a0030632.

Vallerand, R.J. (1997). Intrinsic and extrinsic motivation in sport: implications from the hierarchical model. in R. Lidor and M. BarEli (eds), Innovations in sport psychology: linking theory and practice (pp. 45-7). Netanya, International Society of Sports Psychology.

Vallerand, R.J. (2001). A Hierarchical Model of Intrinsic and Extrinsic Motivation in Sport and Exercise. Advances in Motivation in Sport and Exercise. Champaign: Human Kinetics.

Vartanian, L. R., Wharton, C. M., \& Green, E. B. (2012). Appearance vs. health motives for exercise and for weight loss. Psychology of Sport $\mathcal{E}$ Exercise, 13, 251-256.

Wang, J., \& Biddle, S. (2003). intrinsic motivation towards sport in Singaporean students: The role of sport ability beliefs. Journal of Health Psychology. 8(5), 515 -523.

Wilson, P. M., Mack, D. E., \& Grattan, K. P. (2008). Understanding motivation for exercise: A self-determination theory perspective. Canadian Psychology /Psychologie Canadienne, 49(3), 250-256.

Doi:10.1037/a0012762

Wilson, P., Rodgers, W., \& Fraser, S. (2002). Examining the psychometric properties of the behavioral regulation in exercise questionnaire. Measurement in Physical Education and Exercise Science, 6, 1-21.

Wilson, P., Rodgers, W., Blanchard, C., \& Gessell, J. (2003). The relationship between psychological needs, self-determined motivation, exercise attitudes, and physical fitness. Journal of Applied Social Psychology, 33, 2373-2392. 